02

\title{
Разработка топологий тонкопленочных СКВИД-датчиков для сверхчувствительных измерений магнитных полей
}

\author{
(C) Е.А. Костюрина, К.В. Калашников, Л.В. Филиппенко, В.П. Кошелец \\ Институт радиотехники и электроники им. В.А. Котельникова РАН, \\ Москва, Россия \\ E-mail: kostyurina@hitech.cplire.ru
}

Разработана и оптимизирована топология тонкопленочных СКВИД-датчиков на базе туннельных переходов $\mathrm{Nb} / \mathrm{AlO}_{x} / \mathrm{Nb}$ для создания на их основе систем неразрушающего контроля материалов и других диагностических систем, обладающих чувствительностью по магнитному полю $<10 \mathrm{fT} / \mathrm{Hz}^{1 / 2}$.

Исследование выполнено при финансовой поддержке Российского научного фонда (проект № 15-19-00206).

Системы на основе сверхпроводниковых квантовых интерференционных устройств (СКВИД) прекрасно зарекомендовали себя в области сверхчувствительных измерений слабых магнитных полей. Одним из перспективных направлений использования магнитометров на базе СКВИД-датчиков является создание на их основе систем неразрушающего контроля материалов и металлических конструкций $[1,2]$, в которых необходима чувствительность по магнитному полю $<10 \mathrm{fT} / \mathrm{Hz}^{1 / 2}$ с уровнем пространственного разрешения дефектов менее $1 \mathrm{~mm}$ в составных металлических структурах толщиной до $15 \mathrm{~mm}$.

Основой двухконтактного СКВИД является сверхпроводящее кольцо с двумя параллельно включенными джозефсоновскими переходами. Принцип работы СКВИД основан на регистрации выходного напряжения $V_{\text {out }}$ (либо тока в соответствующем режиме измерения), индуцированного магнитным потоком, проходящим через петлю СКВИД с коэффициентом преобразования $\partial V_{\text {out }} / \partial \Phi_{\text {SQUID }}>10^{10} \mathrm{~V} / \mathrm{Wb}$, связывающим входной магнитный поток в СКВИД и выходное напряжение на сигнальной характеристике $V_{\text {out }}\left(\Phi_{\text {SQUID }}\right)$ [3].

Измерение магнитных полей с помощью СКВИД требует безгистерезисности его ВАХ, что соответствует значениям параметра МакКамбера $\beta_{c}=$ $=(2 e / h) I_{c} C R^{2}<1$, где $I_{c}, C$ и $R-$ критический ток, емкость и полное сопротивление перехода соответственно. Кроме того, оптимальная глубина модуляции вольтамперной характеристики СКВИД реализуется при значении безразмерной индуктивности кольца СКВИД $\beta_{L}=2 L I_{c} / \Phi_{0} \sim 1$, где $L-$ индуктивность кольца, а $\Phi_{0}$ - квант магнитного потока [4]. При этом характерное напряжение $V_{c}=I_{c} R$ должно быть не менее $100 \mu \mathrm{V}$. Разработке и оптимизации топологии СКВИД-детектора с интегрированной входной катушкой с чувствительностью не хуже $0.4 \mu \mathrm{A} / \Phi_{0}$, обладающего характерным напряжением не менее $100 \mu \mathrm{V}$ и собственными шумами не хуже $10 \mu \Phi_{0} / \mathrm{Hz}^{1 / 2}$, посвящена настоящая работа.

Традиционные топологии СКВИД-детекторов типа „шайба“ обладают рядом недостатков, таких как боль- шая паразитная индуктивность щели, высокая чувствительность к паразитным магнитным полям и сильное влияние шумов токов смещения на детектор. Для повышения чувствительности СКВИД-датчика, а также уменьшения его собственных шумов была разработана высокосимметричная конструкция, позволяющая преодолеть перечисленные недостатки.

Топологически микросхема СКВИД-детектора представляет собой сложную тонкопленочную многослойную структуру, состоящую из шести основных слоев (рис. 1). На подложке из монокристаллического кремния с защитным слоем из $\mathrm{Al}_{2} \mathrm{O}_{3}$ формируется базовый электрод $M 1$, представляющий собой симметричное соединение под углом $90^{\circ}$ четырех структур классической геометрии типа „шайба“. Такая конструкция представляет собой прямоугольную пленку из ниобия, в центре которой соединяются четыре щели под углом $90^{\circ}$. На концах щелей расположены круглые отверстия, два из которых индуктивно связаны с входными катушками, а оставшиеся два - с катушками модуляции. Размеры отверстий и длина щели определяют индуктивность петли СКВИД, оптимальное значение которой для критического тока одного перехода 6-12 $\mu$ А составляет около $70-140 \mathrm{pH}$, что соответствует оптимальной глубине модуляции вольт-амперной характеристики СКВИД.

Два джозефсоновских перехода формируются в непосредственной близости от пересечения щелей (по разные стороны от него). Площади переходов равны друг другу и составляют $1-2 \mu \mathrm{m}^{2}$ для разных вариантов образцов. Переходы формировались из трехслойной структуры $\mathrm{Nb} / \mathrm{AlO}_{x} / \mathrm{Nb}$ методом Selective Niobium Etching and Anodization Process [5]; в результате нижняя пленка ниобия и торцы покрывались тонкой пленкой оксида ниобия $\mathrm{Nb}_{2} \mathrm{O}_{5}$.

Изготовленные таким образом переходы обладают нормальным сопротивлением $R_{n} \sim 200 \Omega$. Требование безгистерезисности ВАХ приводит к необходимости шунтирования туннельного перехода сверхпроводникизолятор-сверхпроводник (СИС) сопротивлением порядка $10 \Omega$, в качестве которого использовались полоски 


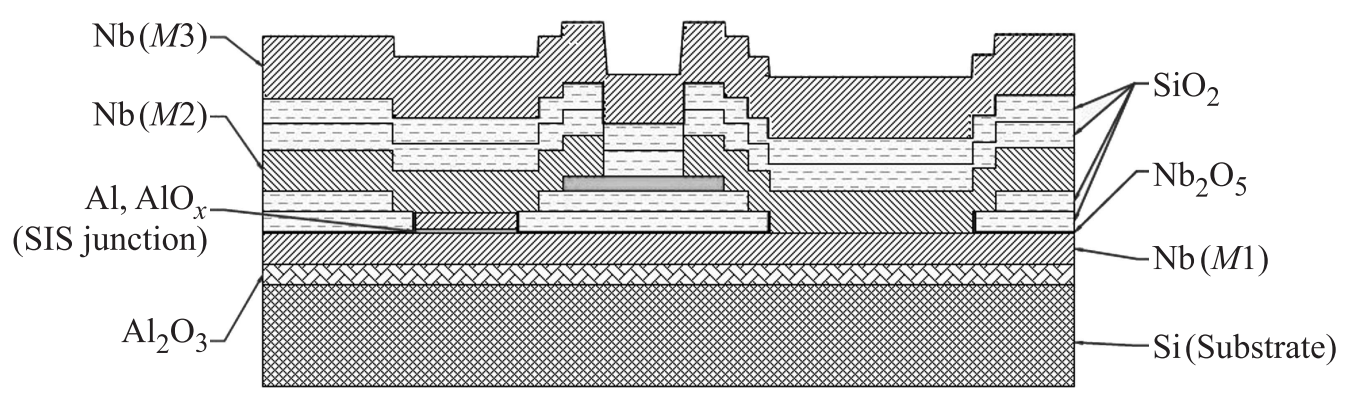

Рис. 1. Схематический разрез микросхемы в области шунтированного СИС-перехода.

молибдена толщиной $70 \mathrm{~nm}$, имеющие сопротивление при $4.2 \mathrm{~K}$ около $2 \Omega / \square$. Межслойное соединение шунта с нижним электродом возможно либо с помощью непосредственного электрического контакта, либо через туннельный переход большой площади. Второй способ проще в реализации, но является менее предпочтительным, поскольку в этом случае при частотах, близких к плазменной частоте СИС-переходов, ВАХ имеет особенности, затрудняющие работу СКВИД. Поэтому шунтирование реализовывалось посредством прямого соединения шунта с нижним электродом, что потребовало дополнительной технологической операции.

Постоянный ток смещения подается на детектор с помощью четырех диагональных симметрично расположенных дорожек во втором слое металлизации $M 2$ (рис. 2). Высокая симметрия дорожек задания токов смещения и шунтирующих элементов обеспечивает взаимную компенсацию создаваемых ими магнитных потоков, а также уменьшает влияние текущих через переход токов как на петлю СКВИД, так и на входные и модуляционные катушки, что делает датчик нечувствительным к токовым флуктуациям.

В одном из вариантов реализации СКВИД-датчика во втором слое металлизации над щелями сформированы сверхпроводящие экраны, позволяющие экранировать магнитное поле токов в кольце СКВИД и тем самым

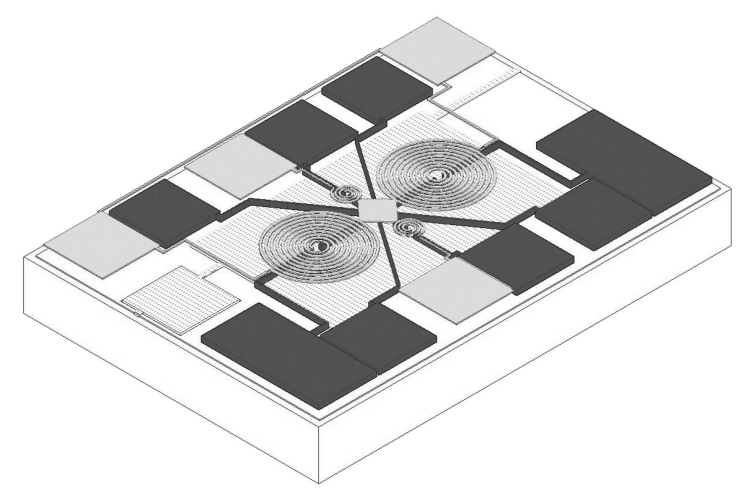

Рис. 2. Топология микросхемы. Показаны слои металлизации: $M 1$ (белый с темными точками), $M 2$ (темно-серый), M3 (светло-серый). значительно уменьшить вклад щелей в индуктивность петли СКВИД. Таким образом, появляется возможность увеличить критический ток перехода примерно в 2 раза с той же расчетной глубиной модуляции.

В третьем слое металлизации $M 3$ сформированы две входные (индуктивность около $0.3-1.3 \mu \mathrm{H}$ ) и две модуляционные $(10 \mathrm{pH})$ катушки с ширинами $w$ и межвитковыми расстояниями $s 2.5$ и $5 \mu \mathrm{m}$, индуктивно связанные с отверстиями СКВИД [3,6]. Геометрия расположения входных и модуляционных катушек обеспечивает малую взаимоиндукцию между ними, а направление токов в них выбрано таким образом, чтобы создаваемые ими магнитные потоки были попарно противонаправлены. Также в слое $M 3$ расположен сверхпроводящий экран, уменьшающий индуктивность участка щели, не закрытого катушкой. Отметим, что построенный таким образом СКВИД является градиентометром первого порядка, а следовательно, нечувствителен к внешним постоянным магнитным полям.

По разработанным топологиям датчиков были спроектированы фотошаблоны и изготовлена первая тестовая партия экспериментальных образцов. Для СКВИД с площадью переходов $S \sim 2 \mu \mathrm{m}^{2}$ критический ток $I_{c} \sim 40 \mu \mathrm{A}$, характерное напряжение $I_{c} R \sim 140 \mu \mathrm{V}$, что примерно в 2 раза больше расчетных значений. На рис. 3 представлена ВАХ такого СКВИД-датчика.

Среди проблем, влияющих на корректную работу СКВИД-датчика, можно выделить возникновение резонансов, связанных с геометрией СКВИД. Один из типов резонансов обусловлен возникновением стоячих волн в микрополосковой линии, образованной между входной или модуляционной катушкой и нижним слоем металлизации $M 1$ [7]. При этом резонансные частоты определяются выражением

$$
f_{s}=v / 4 l, \quad v=\left(L_{\text {strip }} C_{\text {strip }}\right)^{-1.2},
$$

где $L_{\text {strip }}$ и $C_{\text {strip }}-$ погонные индуктивность и емкость микрополосковой линии соответственно [8].

В используемой топологии в область рабочих частот попадают резонансы, связанные с катушками модуляции. Они приводят к особенностям, которые можно видеть на вольт-амперной характеристике СКВИД (рис. 3). Для катушки в $N=8$ витков с шагом $10 \mu \mathrm{m}(w=5 \mu \mathrm{m}$, 


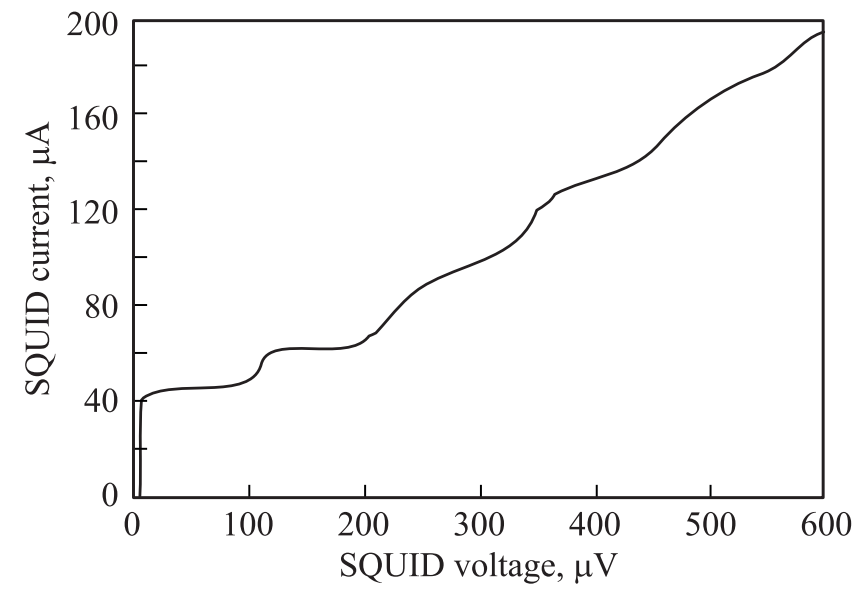

Рис. 3. Вольт-амперная характеристика СКВИД.

$s=5 \mu \mathrm{m})$ частота основной гармоники, вычисленная по данной формуле, оказывается равной $f=47 \mathrm{GHz}$, что соответствует напряжению $V=97 \mu \mathrm{V}$. Из рис. 3 видно, что первая особенность ВАХ с хорошей точностью соответствует полученной частоте. Частоты высших гармоник вычисляются по формуле $f=n f_{s}, n$ - натуральное число. Наличие рассмотренных резонансов приводит к уменьшению рабочей области датчика, а также к обрезанию сигнальной характеристики, представляющей собой зависимость напряжения на переходах от внешнего поля. Повысить резонансные частоты и вывести их тем самым из рабочей области можно, варьируя параметры катушек, например уменьшая количество витков катушек модуляции. Кроме того, резонансы можно существенно демпфировать путем введения дополнительных резистивных элементов $[9,10]$.

Чтобы избавиться от проблемы, связанной с обрезанием сигнальной характеристики резонансами, измерялась зависимость тока через СКВИД от внешнего поля. Это можно сделать, перейдя из режима задания тока в режим задания напряжения. Такая зависимость представлена на рис. 4 (кроме того, такой метод измерений позволяет оценить безразмерную индуктивность СКВИД $\beta_{L}=2 L I_{c} / \Phi_{0}$ как отношение $I_{\max } / \Delta I$, что приблизительно верно для $\left.\beta_{L}>1[3]\right)$.

Еще одной проблемой, связанной с тестированием СКВИД, является большая чувствительность СИС-переходов малой площади к мощным импульсам, вызванным электростатическим разрядом или скачком напряжения питания, что часто приводит к „пробою“ переходов. Для дополнительной защиты образцов было предложено проводить измерения по пятиточечной схеме (рис. 5). Напряжение на СИС-переходах измеряется через контакты 5 и 6 , а ток через переход рассчитывается по падению напряжения на резисторе $R 2\left(V_{\text {sense }}\right)$. Предложенная схема измерений называется пятиточечной, поскольку в реальных системах провода 4 и 5 объединяются в один, что можно сделать, поскольку ток по ним не течет и падение напряжения пренебрежимо мало.
На рис. 4, $a$ изображена сигнальная характеристика одного из СКВИД-датчиков, представляющая собой зависимость тока через СКВИД от внешнего поля. Видно, что данный СКВИД обладает чувствительностью по входу $0.3 \mu \mathrm{A} / \Phi_{0}$.

Собственные шумы данного СКВИД составили $\sim 13 \mu \Phi_{0} / \mathrm{Hz}^{1 / 2}$, что уже достаточно для применения в системах неразрушающего контроля материалов. Для подавления паразитных резонансов, вызываемых высокодобротными сверхпроводниковыми линиями над
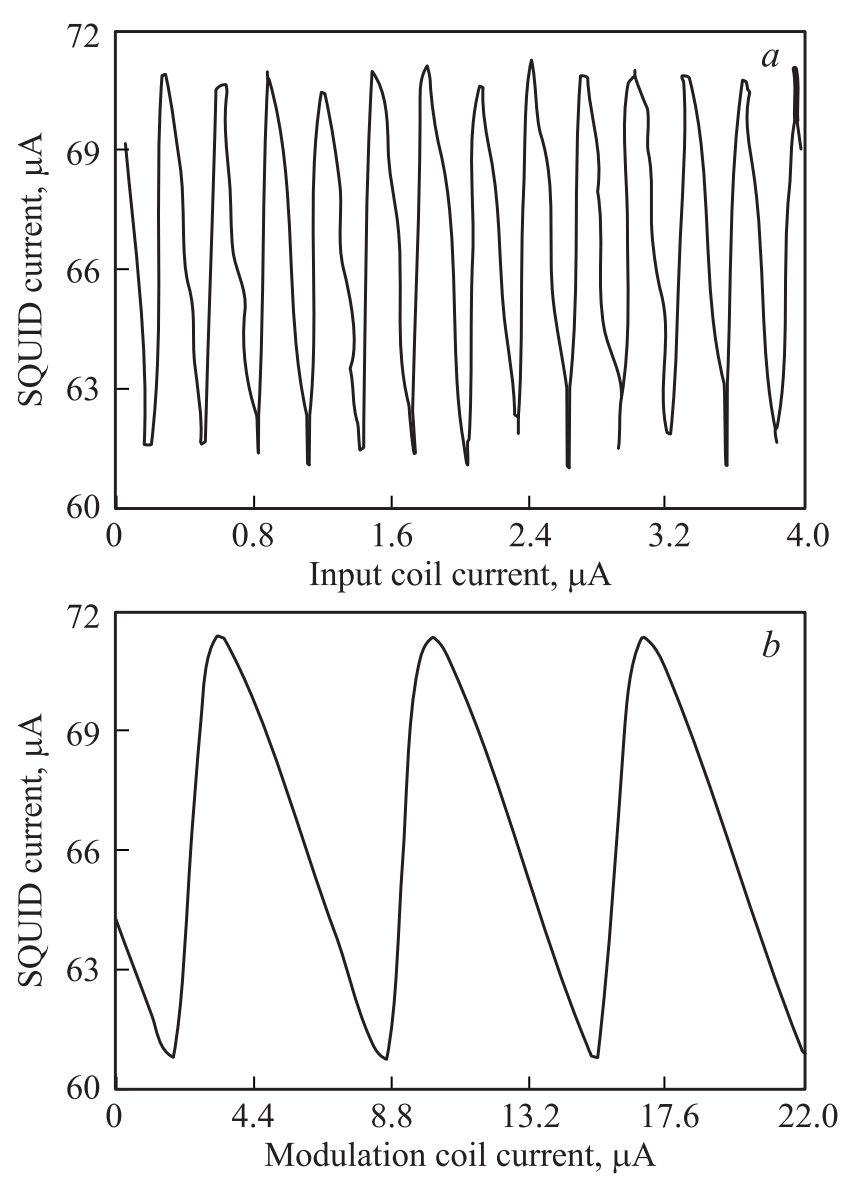

Рис. 4. Сигнальная характеристика СКВИД при задании тока через входную катушку $(a)$ и катушку модуляции $(b)$.

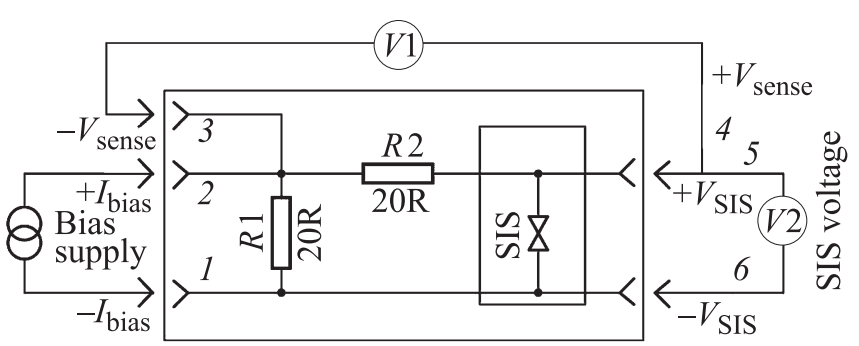

Рис. 5. Пятиточечная схема подключения образца. 1, 2 - контакты задания тока, 3, 4 - дополнительный контакт для измерения напряжения, 5, 6- контакты для измерения напряжения на СИС-переходе. 


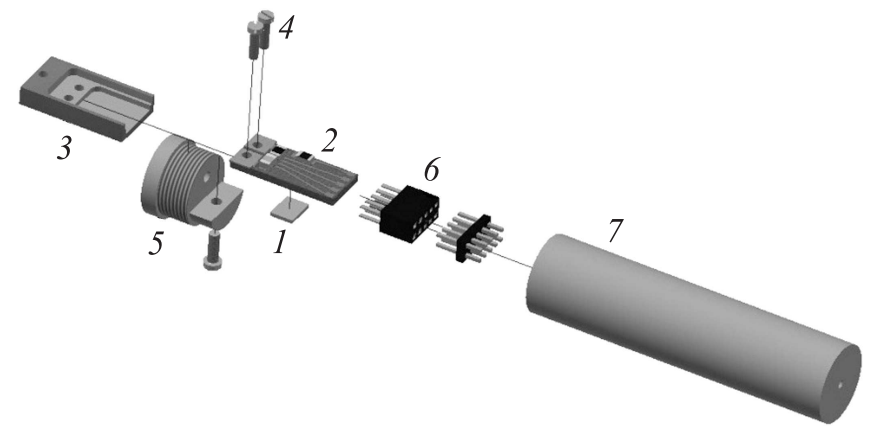

Рис. 6. Схема капсулирования СКВИД-датчика: 1 - микросхема датчика, 2 - стеклотекстолитовая плата, 3 - крышка, 4 - винты, 5 - Nb-держатель, 6 - разъемы для задания токов смещения, $7-\mathrm{Nb}$-экран.

экраном, в оптимизированной версии интегрального СКВИД-датчика будут модифицированы катушки модуляции и связи, а также использованы дополнительные резистивные элементы, которые смогут демпфировать нежелательные резонансы. Подробные результаты измерения шумов новой версии СКВИД-датчика будут представлены в отдельной работе.

Для использования СКВИД-датчиков в практических приложениях была разработана монтажная конструкция для соединения микросхемы с внешней электроникой, установки дополнительных электрических компонентов и защиты датчика от механических повреждений. В данной установке микросхема СКВИД-детектора закрепляется на текстолитовой плате с помощью эпоксидного клея, после чего контактные площадки токов смещения и катушек модуляции соединяются с помощью ультразвуковой сварки тонкой алюминиевой проволоки диаметром $25 \mu \mathrm{m} \mathrm{c}$ соответствующими площадками на плате, выходящими на разъем $2 \times 5$ типа PBD-10. Провода к ответной части разъема подходят в виде витых пар, объединяющих соответствующие вводы (например, „Плюс“ и „минус“ токов смещения), что также делает датчик более устойчивым к электромагнитным помехам в цепях управляющей электроники. Схема капсулирования СКВИД-датчика приведена на рис. 6. На стеклотекстолитовой плате расположен резистор номиналом $1 \mathrm{k} \Omega$ для отогревания образца, а также фильтр нижних частот. Фильтр образован последовательным соединением конденсатора емкостью $2.5 \mathrm{nF}$ и резистора сопротивлением $39 \Omega$ и подключен параллельно с входной и измерительной катушками.

Таким образом, нами разработана и оптимизирована топология СКВИД-датчика, обладающего чувствительностью входной катушки $\sim 0.3 \mu \mathrm{A} / \Phi_{0}$, характерным напряжением $V \sim 150 \mu \mathrm{V}$ и собственными шумами $\sim 1.3 \cdot 10^{-5} \Phi_{0} / \mathrm{Hz}^{1 / 2}$. Проведено экспериментальное исследование разработанных образцов. Обнаружено, что в них присутствуют резонансы, мешающие корректной работе СКВИД. Определены шаги по дальнейшей оптимизации датчиков с целью использования их в си- стемах для сверхчувствительных измерений магнитных полей, таких как системы неразрушающего контроля материалов.

\section{Список литературы}

[1] W.G. Jenks, S.S.H. Sadeghi, J.P. Wikswo Jr. J. Phys. D 30, 293 (1997).

[2] E.V. Burmistrov. J. Commun. Technol. Electron. 51, 1319 (2006).

[3] The SQUID handbook / Eds J. Clarke, A.I. Braginski. WileyVCH Verlag, Weinheim (2004). 395 p.

[4] C.D. Tesche, J. Clarke. J. Low. Temp. Phys. 27, 301 (1977).

[5] L.V. Filippenko, S.V. Shitov, P.N. Dmitriev, A.B. Ermakov, V.P. Koshelets, J.R. Gao. IEEE Trans. Appl. Supercond. 11, 816 (2001).

[6] J. Knuutila, M. Kajola, H. Seppä, R. Mutikainen, J. Salmi. J. Low. Temp. Phys. 71, 369 (1988).

[7] K. Enpuku, R. Cantor, H. Koch. J. Appl. Phys. 72, 1000 (1992).

[8] W.H. Chang. J. Appl. Phys. 50, 8129 (1979).

[9] J. Knuutila, A. Ahonen, C. Tesche. J. Low. Temp. Phys. 68, 269 (1987).

[10] M.E. Huber, A.H. Steinbach, R.H. Ono. Physica C 351, 85 (2001). 